\title{
Computing Minimal Doubly Resolving Sets and the Strong Metric Dimension of the Layer Sun Graph and the Line Graph of the Layer Sun Graph
}

\author{
Jia-Bao Liu $\mathbb{D i D}^{1,2}$ and Ali Zafari $\mathbb{D D}^{3}$ \\ ${ }^{1}$ School of Finance and Mathematics, Huainan Normal University, Huainan 232038, China \\ ${ }^{2}$ School of Mathematics and Physics, Anhui Jianzhu University, Hefei 230601, China \\ ${ }^{3}$ Department of Mathematics, Faculty of Science, Payame Noor University, P.O. Box 19395-4697, Tehran, Iran \\ Correspondence should be addressed to Ali Zafari; zafari.math.pu@gmail.com
}

Received 1 June 2020; Accepted 10 September 2020; Published 24 September 2020

Academic Editor: Lucia Valentina Gambuzza

Copyright ( 2020 Jia-Bao Liu and Ali Zafari. This is an open access article distributed under the Creative Commons Attribution License, which permits unrestricted use, distribution, and reproduction in any medium, provided the original work is properly cited.

\begin{abstract}
Let $G$ be a finite, connected graph of order of, at least, 2 with vertex set $V(G)$ and edge set $E(G)$. A set $S$ of vertices of the graph $G$ is a doubly resolving set for $G$ if every two distinct vertices of $G$ are doubly resolved by some two vertices of $S$. The minimal doubly resolving set of vertices of graph $G$ is a doubly resolving set with minimum cardinality and is denoted by $\psi(G)$. In this paper, first, we construct a class of graphs of order $2 n+\sum_{r=1}^{k-2} \mathrm{~nm}^{r}$, denoted by LSG $(n, m, k)$, and call these graphs as the layer Sun graphs with parameters $n, m$, and $k$. Moreover, we compute minimal doubly resolving sets and the strong metric dimension of the layer Sun graph LSG $(n, m, k)$ and the line graph of the layer Sun graph LSG $(n, m, k)$.
\end{abstract}

\section{Introduction}

In this paper, suppose $G$ is a finite, simple connected graph of order of, at least, 2, with vertex set $V(G)$ and edge set $E(G)$. If $x$ and $y$ are vertices in the graph $G$, then the distance $x$ from $y$ in $G$ is denoted by $d_{G}(x, y)$ or simply $d(x, y)$, where $d(x, y)$ is the length of the shortest path from $x$ to $y$. The line graph of a graph $G$ is denoted by $L(G)$, with vertex set $V(L(G))=E(G)$ and where two edges of $G$ are adjacent in $L(G)$ if and only if they are incident in $G$, see [1]. Vertices $x, y$ of the graph $G$ are said to doubly resolve vertices $u, v$ of $G$ if $d(u, x)-d(u, y) \neq d(v, x)-d(v, y)$. A set $S$ of vertices of the graph $G$ is a doubly resolving set of $G$ if every two distinct vertices of $G$ are doubly resolved by some two vertices of $S$. The minimal doubly resolving set of vertices of graph $G$ is a doubly resolving set with minimum cardinality and is denoted by $\psi(G)$. The notion of a doubly resolving set of vertices of the graph $G$ was introduced by Cáceres et al. [2]. A vertex $w$ strongly resolves two vertices $u$ and $v$ if $u$ belongs to a shortest $v-w$ path or $v$ belongs to a shortest $u-w$ path. A vertex set $S$ of the graph $G$ is a strong resolving set of $G$ if every two distinct vertices of $G$ are strongly resolved by some vertex of $S$. A strong metric basis of $G$ is denoted by $\operatorname{sdim}(G)$ defined as the minimum cardinality of a strong resolving set of $G$. The notion of a strong metric dimension problem set of vertices of the graph $G$ was introduced by A. Sebö and E. Tannier [3] and further investigated by $O$. R. Oellermann and Peters-Fransen [4]. The minimal doubly resolving sets for jellyfish and cocktail party graphs have been obtained in [5]. For more results related to these concepts, see [6-16]. In this paper, first, we construct a class of graphs of order $2 n+\sum_{r=1}^{k-2} \mathrm{~nm}^{r}$, denoted by LSG $(n, m, k)$, and call these graphs as the layer Sun graphs with parameters $n, m$, and $k$, which is defined as follows:

Let $n, m, k$ be integers such that $n, k \geq 3, m \geq 2$ and $G$ be a graph with vertex set $V(G)=V_{1} \cup V_{2} \cup \ldots \cup V_{k}$, where $V_{1}, V_{2}, \ldots V_{k}$ are called the layers of $G$ such that $V_{1}=V\left(C_{n}\right)=\{1,2, \ldots, n\}, \quad V_{2}=\left\{v_{1}, v_{2}, \ldots, v_{n}\right\}$, and for $l \geq 3$, we have $V_{l}=\left\{B_{1_{1}}^{(l)}, B_{1_{2}}^{(l)}, \ldots, B_{1_{l^{l}-3}}^{(l)} ; B_{2_{1}}^{(l)}, B_{2_{2}}^{(l)}, \ldots\right.$, $\left.B_{2^{l}-3}^{(l)} ; \ldots ; B_{n_{1}}^{(l)}, B_{n_{2}}^{(l)}, \ldots, B_{n_{m l-3}}^{(l)}\right\}$, and let $B_{\mathrm{ij}}^{(l)}=\left\{\bigcup_{t=1}^{m}\left(v_{\mathrm{ij}}, t\right)^{l}\right\}$, such that every $\left(v_{\mathrm{ij}}, t\right)^{l}$ is a vertex in the layer $V_{l}$, and $B_{\mathrm{ij}}^{(l)} \cong$ 
$\overline{K_{m}}$ in the layer $V_{l}, 1 \leq i \leq n, 1 \leq j \leq m^{l-3}, 1 \leq t \leq m$, where $\overline{K_{m}}$ is the complement of the complete graph on $m$ vertices. Now, suppose that every vertex $i$ in the cycle $C_{n}$ is adjacent to exactly one vertex in the layer $V_{2}$, say $v_{i} \in V_{2}$, and every vertex $v_{i}$ in the layer $V_{2}$ is adjacent to exactly $m$ vertices $\left(v_{i 1}, 1\right)^{3},\left(v_{i 1}, 2\right)^{3}, \ldots,\left(v_{i 1}, m\right)^{3} \in B_{i 1}^{(3)} \in V_{3}$, in particular for $l \geq 3$, every vertex $\left(v_{\mathrm{ir}}, t\right)^{l} \in B_{i_{r}}^{(l)} \in V_{l}$ is adjacent to exactly $m$ vertices $\bigcup_{t=1}^{m}\left(v_{i j}, t\right)^{l+1} \in B_{i_{j}}^{(l+1)} \in V_{l+1}$, and then, the resulting graph is called the layer Sun graph $\operatorname{LSG}(n, m, k)$ with parameters $n, m$, and $k$. Also, for $l \geq 3$, we recall that $B_{i_{j}}^{(l)}$ as the components of the layer $V_{l}, 1 \leq i \leq n, 1 \leq j \leq m^{l-3}$. In particular, we say that two components $B_{i_{j}}^{(l)}, B_{r_{s}}^{(l)} \in V_{l}$, $1 \leq i, r \leq n, 1 \leq j, s \leq m^{l-3}$ are fundamental if $i=r$ and $j \neq s$. It is natural to consider its vertex set of the layer Sun graph LSG $(n, m, k)$ as partitioned into $k$ layers. The layers $V_{1}$ and $V_{2}$ consist of the vertices $\{1,2, \ldots, n\}$ and $\left\{v_{1}, v_{2}, \ldots, v_{n}\right\}$, respectively. In particular, each layer $V_{l}(l \geq 3)$ consists of the $\mathrm{nm}^{l-2}$ vertices. Note that, for each vertex $i$ in the layer $V_{1}$ and every vertex $x \in B_{\mathrm{ij}}^{(l)} \in V_{l}, l \geq 3,1 \leq j \leq m^{l-3}$, we have $d(i, x)=l-1$. In this paper, we consider the problem of determining the cardinality $\psi(\operatorname{LSG}(n, m, k))$ of minimal doubly resolving sets of the layer Sun graph $\operatorname{LSG}(n, m, k)$. First, we find the metric dimension of the layer Sun graph $\operatorname{LSG}(n, m, k)$; in fact, we prove that if $n, k \geq 3$ and $m \geq 2$, then the metric dimension of the layer Sun graph $\operatorname{LSG}(n, m, k)$ is $\mathrm{nm}^{k-2}-\mathrm{nm}^{k-3}$. Moreover, we consider the problem of determining the cardinality $\psi(\operatorname{LSG}(n, m, k))$ of minimal doubly resolving sets of $\operatorname{LSG}(n, m, k)$ and the strong metric dimension for the layer Sun graph $\operatorname{LSG}(n, m, k)$ and the line graph of the layer Sun graph $\operatorname{LSG}(n, m, k)$. The graph $\operatorname{LSG}(3,3,4)$ is shown in Figure 1.

\section{Definitions and Preliminaries}

Definition 1. Let $G$ be a graph. A vertex $x \in V(G)$ is said to resolve a pair $u, v \in V(G)$ if $d_{G}(u, x) \neq d_{G}(v, x)$. For an ordered subset $W=\left\{w_{1}, w_{2}, \ldots, w_{k}\right\}$ of vertices in the graph $G$ and a vertex $v$ of $G$, the metric representation of $v$ with respect to $W$ is the $k$-vector $r(v / W)=\left(d\left(v, w_{1}\right)\right.$, $\left.d\left(v, w_{2}\right), \ldots, d\left(v, w_{k}\right)\right)$. If every pair of distinct vertices of $G$ has different metric representations, then the ordered set $W$ is called a resolving set of $G$. If the set $W$ is as small as possible, then it is called a metric basis of the graph $G$. We recall that the metric dimension of $G$, denoted by $\beta(G)$, is defined as the minimum cardinality of a resolving set for $G$.

Proposition 1. Let $G$ be a graph. It is well known that a doubly resolving set is also a resolving set and $\beta(G) \leq \psi(G)$. In particular, every strong resolving set is a resolving set and $\beta(G) \leq \operatorname{sdim}(G)$.

\section{Main Results}

3.1. Minimal Doubly Resolving Sets and the Strong Metric Dimension for the Layer Sun Graph LSG $(n, m, k)$

Theorem 1. Let $G=\operatorname{LSG}(n, m, k)$ be the layer Sun graph which is defined already. Suppose that $n, m, k$ are integers such that $n, k \geq 3$ and $m \geq 2$. Then, the metric dimension of $\operatorname{LSG}(n, m, k)$ is $n m^{k-2}-n m^{k-3}$.

Proof. Let $V(G)=V_{1} \cup V_{2} \cup \ldots \cup V_{k}$, where $V_{1}, V_{2}, \ldots$, $V_{k}$ are called the layers of vertices in the layer Sun graph LSG $(n, m, k)$, which is defined already. It is clear that if $W$ is an ordered subset of the layers $V_{1} \cup V_{2} \cup \ldots \cup V_{k-1}$, then $W$ is not a resolving set in $\operatorname{LSG}(n, m, k)$. We may assume that the layer $V_{k}$ is equal to

$$
\begin{aligned}
V_{k}= & \left\{B_{1_{1}}^{(k)}, B_{1_{2}}^{(k)}, \ldots, B_{1_{m^{k-3}}}^{(k)} ; B_{2_{1}}^{(k)}, B_{2_{2}}^{(k)}, \ldots, B_{2_{m^{k-3}}}^{(k)} ; \ldots ;\right. \\
& \left.B_{n_{1}}^{(k)}, B_{n_{2}}^{(k)}, \ldots, B_{n_{m^{k-3}}}^{(k)}\right\},
\end{aligned}
$$

where $B_{\mathrm{ij}}^{(k)}=\left\{\bigcup_{t=1}^{m}\left(v_{\mathrm{ij}}, t\right)^{k}\right\}, 1 \leq i \leq n, 1 \leq j \leq m^{k-3}$. In the following cases, it can be shown that the metric dimension of the layer Sun graph $\operatorname{LSG}(n, m, k)$ is $\mathrm{nm}^{k-2}-\mathrm{nm}^{k-3}$.

Case 1: let $W$ be an ordered subset of the layer $V_{k}$ in the layer Sun graph $\operatorname{LSG}(n, m, k)$ such that

$$
\begin{aligned}
W= & \left\{B_{1_{2}}^{(k)}, \ldots, B_{1_{m^{k-3}}}^{(k)} ; B_{2_{1}}^{(k)}, B_{2_{2}}^{(k)}, \ldots, B_{2_{m^{k-3}}}^{(k)} ; \ldots ;\right. \\
& \left.B_{n_{1}}^{(k)}, B_{n_{2}}^{(k)}, \ldots, B_{n_{m^{k-3}}}^{(k)}\right\} .
\end{aligned}
$$

Hence,

$$
V(G)-W=\left\{V_{1}, V_{2}, \ldots, V_{k-1}, B_{1_{1}}^{(k)}\right\} .
$$

We know that the cardinality of $W$ is $n m^{k-2}-m$ because $\left|B_{i_{j}}^{(k)}\right|=m 1 \leq i \leq n, 1 \leq j \leq m^{k-3}$. Therefore, the metric representation of all the vertices $\left(v_{1}, 1\right)^{k},\left(v_{1_{1}}, 2\right)^{k}, \ldots,\left(v_{1_{1}}, m\right)^{k}$ in the component $B_{1_{1}}^{(k)}$ is the same as $\mathrm{nm}^{\mathrm{k}-2}-\mathrm{m}$-vector with respect to $W$. Thus, $W$ is not a resolving set in $\operatorname{LSG}(n, m, k)$

Case 2: let $W$ be an ordered subset of the layer $V_{k}$ in the layer Sun graph $\operatorname{LSG}(n, m, k)$ such that

$$
\begin{aligned}
W= & \left\{B_{1_{1}}^{(k)}-\left\{\left(v_{1_{1}}, 1\right)^{k},\left(v_{1_{1}}, 2\right)^{k}\right\}, B_{1_{2}}^{(k)}, \ldots, B_{1_{m^{k-3}}}^{(k)} ;\right. \\
& \left.B_{2_{1}}^{(k)}, B_{2_{2}}^{(k)}, \ldots, B_{2_{m^{k-3}}}^{(k)} ; \ldots ; B_{n_{1}}^{(k)}, B_{n_{2}}^{(k)}, \ldots, B_{n_{m^{k-3}}}^{(k)}\right\} .
\end{aligned}
$$

Hence,

$$
V(G)-W=\left\{V_{1}, V_{2}, \ldots, V_{k-1},\left(v_{1_{1}}, 1\right)^{k},\left(v_{1_{1}}, 2\right)^{k}\right\} .
$$




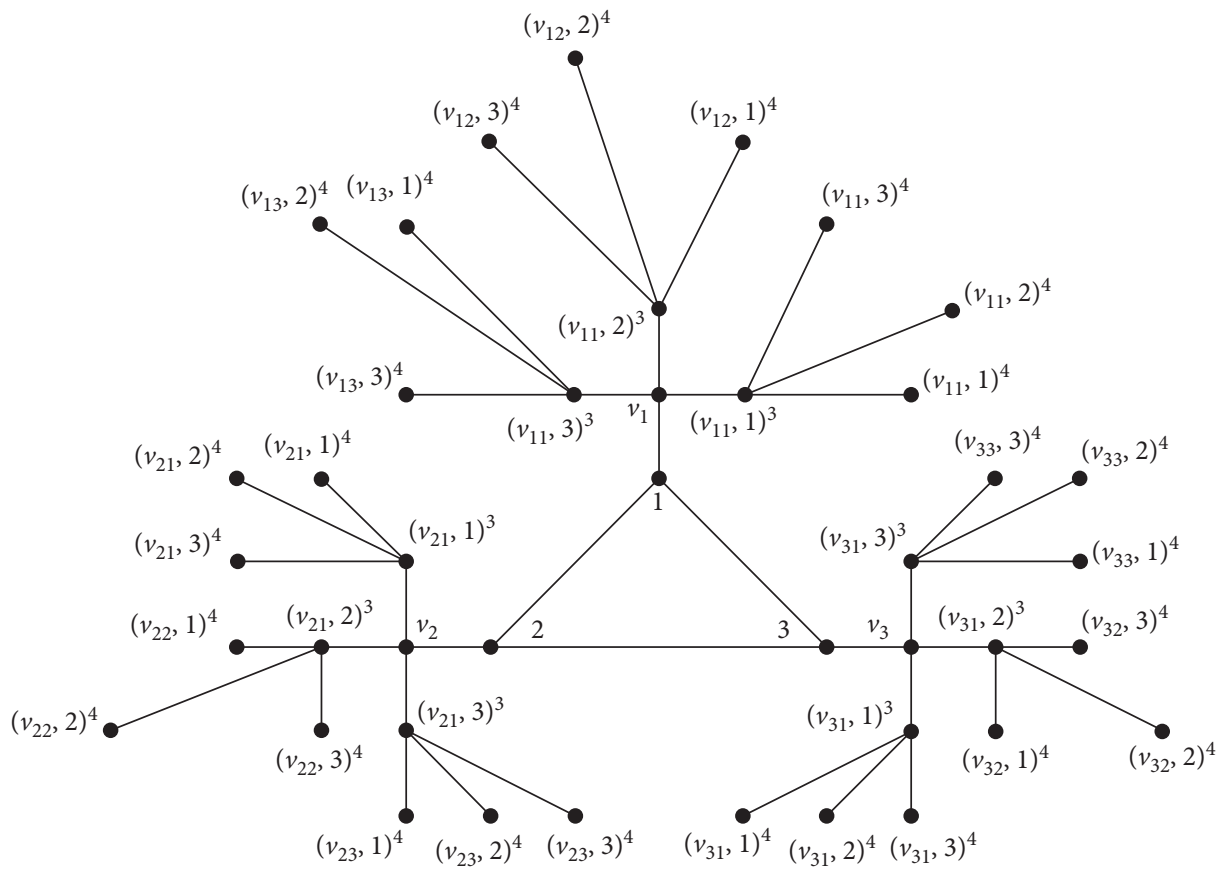

Figure 1: LSG(3,3,4).

We know that $|W|=\mathrm{nm}^{k-2}-2$. Therefore, the metric representation of two vertices $\left(v_{1_{1}}, 1\right)^{k},\left(v_{1_{1}}, 2\right)^{k}$ in the component $B_{1_{1}}^{(k)}$ is the same as $n^{k-2}-2$-vector with respect to $W$. Thus, $W$ is not a resolving set in $\operatorname{LSG}(n, m, k)$.

Case 3: let $W$ be an ordered subset of the layer $V_{k}$ in the layer Sun graph $\operatorname{LSG}(n, m, k)$ such that

$$
\begin{aligned}
W= & \left\{B_{1_{1}}^{(k)}-\left(v_{1_{1}}, 1\right)^{k}, B_{1_{2}}^{(k)}-\left(v_{1_{2}}, 1\right)^{k}, \ldots, B_{1_{m^{k-3}}}^{(k)}\right. \\
& -\left(v_{1_{m^{k-3}}}, 1\right)^{k} ; \ldots ; B_{n_{1}}^{(k)}-\left(v_{n_{1}}, 1\right)^{k}, B_{n_{2}}^{(k)} \\
& \left.-\left(v_{n_{2}}, 1\right)^{k}, \ldots, B_{n_{m^{k-3}}}^{(k)}-\left(v_{n_{m^{k-3}}}, 1\right)^{k}\right\} .
\end{aligned}
$$

Hence,

$$
\begin{aligned}
V(G)-W= & \left\{V_{1}, V_{2}, \ldots, V_{k-1},\left(v_{1_{1}}, 1\right)^{k}, \ldots,\right. \\
& \left.\left(v_{1_{m^{k-3}}}, 1\right)^{k}, \ldots,\left(v_{n_{1}}, 1\right)^{k}, \ldots,\left(v_{n_{m^{k-3}}}, 1\right)^{k}\right\} .
\end{aligned}
$$

We know that $|W|=\mathrm{nm}^{k-2}-\mathrm{nm}^{k-3}$. We can show that all the vertices in $V(G)-W$ have different representations with respect to $W$. Let $u$ be the vertex of the layer $V_{1}=V\left(C_{n}\right)=\{1,2, \ldots, n\}$. We can assume without loss of generality that $u=i, 1 \leq i \leq n$. Hence, $d\left(u,\left(v_{i_{j}}, t\right)^{k}\right)=k-1$, where $\left(v_{i_{j}}, t\right)^{k} \in B_{i_{j}}^{(k)}, 1 \leq t \leq m$, $1 \leq j \leq m^{k-3}$; otherwise, if $u \neq i$, then $d\left(u,\left(v_{i}, t\right)^{k}\right)>k-1$. Now, let $u \in V_{2}=\left\{v_{1}, v_{2}, \ldots, v_{n}\right\}$. We can assume without loss of generality that $u=v_{i}$, $1 \leq i \leq n$. Hence, $d\left(u,\left(v_{i}, t\right)^{k}\right)=k-2$, where $\left(v_{i_{j}}, t\right)^{k} \in B_{i_{j}}^{(k)}, \quad 1 \leq t \leq m, \quad 1 \leq j \leq m^{k-3}$; $\quad$ otherwise, if $u \neq v_{i}$, then $d\left(u,\left(v_{i}, t\right)^{k}\right)>k-2$. In a similar way, we can show that all the vertices in the layers $V_{3}, \ldots, V_{k-1}$ have different representations with respect to $W$. In particular, for every vertex $u \in\left\{\left(v_{1_{1}}, 1\right)^{k}\right.$, $\left.\ldots,\left(v_{1^{k-3}}, 1\right)^{k}, \ldots,\left(v_{n_{1}}, 1\right)^{k}, \ldots,\left(v_{n_{m^{k-3}}}, 1\right)^{k}\right\}$, we have $d\left(u,\left(v_{i_{j}}, t\right)^{k}\right)=2,2 \leq t \leq m$, if $u=\left(v_{i_{j}}, 1\right)^{k}$; otherwise, if $u \in\left\{\left(v_{1_{1}}, 1\right)^{k}, \ldots,\left(v_{1_{m^{k-3}}}, 1\right)^{k}, \ldots,\left(v_{n_{1}}, 1\right)^{k}, \ldots,\left(v_{n_{m^{k-3}}}\right.\right.$, $\left.1)^{k}\right\}$ and $u \neq\left(v_{i_{j}}, 1\right)^{k}$, then $d\left(u,\left(v_{i_{j}}, t\right)^{k}\right)>4$. Therefore, all the vertices in $V(G)-W$ have different representations with respect to $W$. This implies that $W$ is a resolving set in $\operatorname{LSG}(n, m, k)$. From the abovementioned cases, we can be concluded that the minimum possible cardinality of a resolving set in $\operatorname{LSG}(n, m, k)$ is $\mathrm{nm}^{k-2}-\mathrm{nm}^{k-3}$.

Theorem 2. Let $G=\operatorname{LSG}(n, m, k)$ be the layer Sun graph which is defined already. Suppose that $n, m, k$ are integers such that $n, k \geq 3$ and $m \geq 2$. Then, the cardinality of minimum doubly resolving set of the $\operatorname{LSG}(n, m, k)$ is $n m^{k-2}$.

Proof. In the following cases, it can be shown that the cardinality of minimum doubly resolving set of the layer Sun graph $\operatorname{LSG}(n, m, k)$ is $\mathrm{nm}^{k-2}$.

Case 1: we know that the ordered subset $W$ of vertices in equation (8) in the layer $V_{k}$ of $\operatorname{LSG}(n, m, k)$ is a resolving set for $\operatorname{LSG}(n, m, k)$ of cardinality $\mathrm{nm}^{k-2}-\mathrm{nm}^{k-3}$. We show that this subset is not a doubly resolving set for 
LSG $(n, m, k)$. Because if the vertex $u=\left(v_{i_{j}}, 1\right.$ )$^{k} \in B_{i_{j}}^{(k)} \in V_{k}, 1 \leq i \leq n, 1 \leq j \leq m^{k-3}$ is adjacent to a vertex $v \in V_{k-1}$, then for every $x, y \in W$, we have $d(u, x)-d(u, y)=d(v, x)-d(v, y)$.

$$
\begin{aligned}
W= & \left\{B_{1_{1}}^{(k)}-\left(v_{1_{1}}, 1\right)^{k}, B_{1_{2}}^{(k)}-\left(v_{1_{2}}, 1\right)^{k}, \ldots, B_{1_{m^{k-3}}}^{(k)}\right. \\
& -\left(v_{1_{m^{k-3}}}, 1\right)^{k} ; \ldots ; B_{n_{1}}^{(k)}-\left(v_{n_{1}}, 1\right)^{k}, B_{n_{2}}^{(k)} \\
& \left.-\left(v_{n_{2}}, 1\right)^{k}, \ldots, B_{n_{m^{k-3}}}^{(k)}-\left(v_{n_{m^{k-3}}}, 1\right)^{k}\right\} .
\end{aligned}
$$

Case 2: now, let the subset of vertices in $\operatorname{LSG}(n, m, k)$ be $W=\left\{B_{1_{1}}^{(k)}-\left(v_{1_{1}}, 1\right)^{k}, B_{1_{2}}^{(k)}, \ldots, B_{1_{m^{k-3}}}^{(k)}, \ldots, B_{n_{1}}^{(k)}, B_{n_{2}}^{(k)}, \ldots, B_{n_{m^{k-3}}}^{(k)}\right\}$.

In a similar fashion as in Case 3 of Theorem 1, we can show that all the vertices in the layers $V_{1}, V_{2}, \ldots, V_{k-1}$ of $\operatorname{LSG}(n, m, k)$ and the vertex $\left(v_{1_{1}}, 1\right)^{k}$ in the layer $V_{k}$ of $\operatorname{LSG}(n, m, k)$ have different representations with respect to $W$. So, $W$ is a resolving set in $\operatorname{LSG}(n, m, k)$ of cardinality $\mathrm{nm}^{k-2}-1$. Note that, in this case, by a similar way as in Case 1, we can show that this subset is not a doubly resolving set for $\operatorname{LSG}(n, m, k)$.

Case 3: finally, let the subset of vertices in $\operatorname{LSG}(n, m, k)$ be

$W=\left\{B_{1_{1}}^{(k)}, B_{1_{2}}^{(k)}, \ldots, B_{1_{m^{k-3}}}^{(k)} ; \ldots ; B_{n_{1}}^{(k)}, B_{n_{2}}^{(k)}, \ldots, B_{n_{m^{k-3}}}^{(k)}\right\}$.

In a similar fashion as in Theorem 1 , we can show that all the vertices in the layers $V_{1}, V_{2}, \ldots, V_{k-1}$ of $\operatorname{LSG}(n, m, k)$ have different representations with respect to $W$. So, this subset is also a resolving set in $\operatorname{LSG}(n, m, k)$ of cardinality $\mathrm{nm}^{k-2}$. We show that this subset is a doubly resolving set for $\operatorname{LSG}(n, m, k)$. It is sufficient to prove that for two vertices $u$ and $v$ in $\operatorname{LSG}(n, m, k)$, there are vertices $x, y \in W$ such that $d(u, x)-d(u, y) \neq d(v, x)-d(v, y)$. Consider two vertices $u$ and $v$ in $\operatorname{LSG}(n, m, k)$. Then, we have the following.

Case 3.1: suppose that both vertices $u$ and $v$ lie in the layer $V_{1}$. Hence, there are $r, s \in\{1,2, \ldots, n\}$ such that $u=r$ and $v=s$. Moreover, we know that the layer Sun graph $\operatorname{LSG}(n, m, k)$ has the property that, for each vertex $r$ in the layer $V_{1}$, there is some vertex such as $x=\left(v_{r_{j}}, t\right)^{k}$ in the component $B_{r_{j}}^{(k)}, 1 \leq j \leq m^{k-3}$ in the layer $V_{k}$, at distance $k-1$ from $u$; in fact, $d(u, x)=k-1$. In the same way, there is some vertex such as $y=\left(v_{s_{j}}, t\right)^{k}$ in the component $B_{s_{j}}^{(k)}$, in the layer $V_{k}$, at distance $k-1$ from $v$. In particular, it is easy to prove that $d(u, x)-d(u, y)<0$ because $d(u, y) \geq k$. Also, $d(v, x)-d(v, y)>0$ because $d(v, x) \geq k$.

Case 3.2: now, suppose that both vertices $u$ and $v$ lie in the layer $V_{2}$. In a similar way as in Case 3.1, we can show that there are vertices $x, y \in W$ such that $d(u, x)-d(u, y) \neq d(v, x)-d(v, y)$.
Case 3.3: suppose that both vertices $u$ and $v$ lie in the layer $V_{l}, l \geq 3$ such that these vertices lie in the one component of the layer $V_{l}$, say $B_{i_{j}}^{(l)}, 1 \leq i \leq n$, $1 \leq j \leq m^{l-3}$. In this case, $d(u, v)=2$. Moreover, we know that the layer Sun graph LSG $(n, m, k)$ has the property that, for each vertex $u \in B_{i_{j}}^{(l)}$ in the layer $V_{l}$, there is a component of the layer $V_{k}$, say $B_{i_{r}}^{(k)}, 1 \leq i \leq n$, $1 \leq r \leq m^{k-3}$ such that for any vertex $x \in B_{i_{r}}^{(k)}$, we have $d(u, x)=k-l$. In the same way, for the vertex $v \in B_{i_{j}}^{(l)}$ in the layer $V_{l}$, there is a component of the layer $V_{k}$, say $B_{i_{s}}^{(k)}, 1 \leq i \leq n, 1 \leq s \leq m^{k-3}, r \neq s$ such that, for any vertex $y \in B_{i_{s}}^{(k)}$, we have $d(v, y)=k-l$. Thus, $d(u, x)-d(u, y) \neq d(v, x)-d(v, y)$ because $d(u, y)=k-l+2$ and $d(v, x)=k-l+2$.

Case 3.4: suppose that both vertices $u$ and $v$ lie in the layer $V_{l}, l \geq 3$ such that these vertices lie in the two distinct components of the layer $V_{l}$. We can assume without loss of generality that $u \in B_{p_{j_{1}}}^{(l)}$ and $v \in B_{q_{j_{2}}}^{(l)}$, $1 \leq p, q \leq n$, and $1 \leq j_{1}, j_{2} \leq m^{l-3}$. Moreover, we know that the layer Sun graph LSG $(n, m, k)$ has the property that, for each vertex $u \in B_{p_{j_{1}}}^{(l)}$ in the layer $V_{l}$, there is a component of the layer $V_{k}$, say $B_{p_{r}}^{(k)}, 1 \leq r \leq m^{k-3}$ such that for any vertex $x \in B_{p_{r}}^{(k)}$, we have $d(u, x)=k-l$. In the same way, for the vertex $v \in B_{q_{j_{2}}}^{(l)}$ in the layer $V_{l}$, there is a component of the layer $V_{k}$, say $B_{q_{s}}^{(k)}$, $1 \leq s \leq m^{k-3}$, such that for any vertex $y \in B_{q_{s}}^{(k)}$, we have $d(v, y)=k-l$. In the following, let two components $B_{p_{j_{1}}}^{(l)}$ and $B_{q_{j_{2}}}^{(l)}$ be fundamental; indeed, $p=q$. Hence, $d(u, v)=2 l-4, d(u, y)=d(v, x)=k+l-4$. Thus, $d(u, x)-d(u, y) \neq d(v, x)-d(v, y)$. Now, let two components $B_{p_{j_{1}}}^{(l)}$ and $B_{q_{j_{2}}}^{(l)}$ not be fundamental; indeed, $p \neq q$. Hence, $d(u, v)=2 l-2+d_{C_{n}}(p, q), d(u, y)=$ $d(v, x)=k+l-2+d_{C_{n}}(p, q)$. Thus, $d(u, x)-d(u$, $y) \neq d(v, x)-d(v, y)$.

Case 3.5: suppose that vertices $u$ and $v$ lie in distinct layers $V_{a}, V_{b}$, respectively. Note that if $a=1$ and $b=2, a=1$ and $b>2$, or $a=2$ and $b>2$, there is nothing to do. Now, let $3 \leq a<b$. Hence, there is a component of the layer $V_{a}$, say $B_{i_{j}}^{(a)}, 1 \leq i \leq n$, $1 \leq j \leq m^{a-3}$ such that $u \in B_{i_{j}}^{(a)}$. Also, there is a component of the layer $V_{b}$, say $B_{p_{q}}^{(b)}, 1 \leq p \leq n$, $1 \leq q \leq m^{b-3}$ such that $v \in B_{p_{q}}^{(b)}$. In particular, there is a component of the layer $V_{k}$, say $B_{i_{r}}^{(k)}, 1 \leq i \leq n$, $1 \leq r \leq m^{k-3}$ such that for any vertex $x \in B_{i_{r}}^{(k)}$, we have $d(u, x)=k-a$. Now, let $i=p$; if we consider $y \in B_{z_{s}}^{(k)}, z \neq i, 1 \leq z \leq n$, and $1 \leq s \leq m^{k-3}$, then we have $d(u, x)-d(u, y) \neq d(v, x)-d(v, y)$. Because $d(u, y)=k+a-2+d_{C_{n}}(i, z), \quad d(v, y)=k+b-2+$ $d_{C_{n}}(i, z)$ and $d(u, x) \neq d(v, x)$. Note that if $i \neq p$, then there is a component of the layer $V_{k}$, say $B_{p_{s}}^{(k)}$, $1 \leq p \leq n, \quad 1 \leq s \leq m^{k-3}$ such that for any vertex $y \in B_{p_{j}}^{(k)}$, we have $d(v, y)=k-b$, and then, we have 
$d(u, x)-d(u, y) \neq d(v, x)-d(v, y)$ because $d(u, x)-$ $d(u, y)<0$ and $d(v, x)-d(v, y)>0$. Thus, from the abovementioned cases, we can conclude that the cardinality of the minimum doubly resolving set of the layer Sun graph $\operatorname{LSG}(n, m, k)$ is $\mathrm{nm}^{k-2}$.

Theorem 3. Let $G=\operatorname{LSG}(n, m, k)$ be the layer Sun graph which is defined already. Suppose that $n, m, k$ are integers such that $n, k \geq 3$ and $m \geq 2$. Then, the strong metric dimension of $\operatorname{LSG}(n, m, k)$ is $n m^{k-2}-1$.

Proof. In the following cases, it can be seen that the cardinality of the minimum strong resolving set of the layer Sun graph LSG $(n, m, k)$ is $\mathrm{nm}^{k-2}-1$.
Case 1: we know that the ordered subset $W$ of vertices in equation (11) in the layer $V_{k}$ of the layer Sun graph $\operatorname{LSG}(n, m, k)$ is a resolving set for $\operatorname{LSG}(n, m, k)$ of cardinality $\mathrm{nm}^{k-2}-\mathrm{nm}^{k-3}$. Now, let $N=V_{k}-W=\left\{\left(v_{1_{1}}, 1\right)^{k}, \ldots,\left(v_{1_{m^{k-3}}}, 1\right)^{k}, \ldots,\left(v_{n_{1}}, 1\right)^{k}\right.$, $\left.\ldots,\left(v_{n_{m^{k-3}}}, 1\right)^{k}\right\}$. By considering distinct vertices $u, v \in N$, we can show that there is not a vertex $w \in W$ such that $u$ belongs to a shortest $v-w$ path or $v$ belongs to a shortest $u-w$ path because the valency of every vertex in the layer $V_{k}$ is one. So, this subset is not a strong resolving set for $G$. Thus, we can be conclude that if $W$ is a strong resolving set for graph $G$, then $|W| \geq \mathrm{nm}^{k-2}-1$ because $|N|$ must be less than 2 .

$$
W=\left\{B_{1_{1}}^{(k)}-\left(v_{1_{1}}, 1\right)^{k}, B_{1_{2}}^{(k)}-\left(v_{1_{2}}, 1\right)^{k}, \ldots, B_{1_{m^{k-3}}}^{(k)}-\left(v_{1_{m^{k-3}}}, 1\right)^{k} ; \ldots ; B_{n_{1}}^{(k)}-\left(v_{n_{1}}, 1\right)^{k}, B_{n_{2}}^{(k)}-\left(v_{n_{2}}, 1\right)^{k}, \ldots, B_{n_{m^{k-3}}}^{(k)}-\left(v_{n_{m^{k-3}}}, 1\right)^{k}\right\},
$$

Case 2: on the other hand, we can show that the subset $W$ of vertices in equation (12) in the graph $G$ is a resolving set for graph $G$. We show that this subset is a strong resolving set in graph $G$. It is sufficient to prove that every two distinct vertices $u, v \in V(G)-W$ are strongly resolved by a vertex $w \in W$. Then, we have the following:

$$
W=\left\{B_{1_{1}}^{(k)}-\left(v_{1_{1}}, 1\right)^{k}, B_{1_{2}}^{(k)}, \ldots, B_{1_{m^{k-3}}}^{(k)}, \ldots, B_{n_{1}}^{(k)}, B_{n_{2}}^{(k)}, \ldots, B_{n_{m^{k-3}}}^{(k)}\right\}
$$

Case 2.1: suppose that both vertices $u$ and $v$ lie in the layer $V_{1}$. Hence, there are $r, s \in\{1,2, \ldots, n\}$ such that $u=r$ and $v=s$. Moreover, we know that the layer Sun graph $\operatorname{LSG}(n, m, k)$ has the property that, for each vertex $r$ in the layer $V_{1}$, there is a component $B_{r}^{(k)}$, $1 \leq j \leq m^{k-3}$ in the layer $V_{k}$ such that, for every vertex such as $w \in B_{r_{j}}^{(k)}$, we have $d(u, w)=k-1$ and $d(v, w)>k-1$, and hence, $u$ belongs to a shortest $w-v$ path.

Case 2.2: now suppose that both vertices $u$ and $v$ lie in the layer $V_{2}$. In a similar way as in Case 2.1, we can show that the vertices $u$ and $v$ are strongly resolved by a vertex $w \in W$.

Case 2.3: suppose that both vertices $u$ and $v$ lie in the layer $V_{l}, l \geq 3$ such that these vertices lie in the one component of the layer $V_{l}$, say $B_{i_{j}}^{(l)}, 1 \leq i \leq n$, $1 \leq j \leq m^{l-3}$. In this case, $d(u, v)=2$. Moreover, we know that the layer Sun graph LSG $(n, m, k)$ has the property that, for each vertex $u \in B_{i_{j}}^{(l)}$ in the layer $V_{l}$, there is a component of the layer $V_{k}$, say $B_{i_{r}}^{(k)}, 1 \leq i \leq n$, $1 \leq r \leq m^{k-3}$ such that for any vertex $w \in B_{i_{r}}^{(k)}$, we have $d(u, w)=k-l$, and hence, $u$ belongs to a shortest $w-v$ path.
Case 2.4: suppose that both vertices $u$ and $v$ lie in the layer $V_{l}, l \geq 3$ such that these vertices lie in the two distinct components of the layer $V_{l}$. We can assume without loss of generality that $u \in B_{p_{i_{1}}}^{(l)}$ and $v \in B_{q_{i_{2}}}^{(l)}$, $1 \leq p, q \leq n$, and $1 \leq j_{1}, j_{2} \leq m^{l-3}$. Moreover, we know that the layer Sun graph LSG $(n, m, k)$ has the property that, for each vertex $u \in B_{p_{i}}^{(l)}$ in the layer $V_{l}$, there is a component of the layer $V_{k}$, say $B_{p_{r}}^{(k)}, 1 \leq r \leq m^{k-3}$ such that for any vertex $w \in B_{p_{r}}^{(k)}$, we have $d(u, w)=k-l$, and hence, $u$ belongs to a shortest $w-$ $v$ path.

Case 2.5: suppose that vertices $u$ and $v$ lie in distinct layers $V_{a}, V_{b}$, respectively. Note that if $a=1$ and $b=2, a=1$ and $b>2$, or $a=2$ and $b>2$, there is nothing to do. Now, let $3 \leq b<a$. Hence, there is a component of the layer $V_{a}$, say $B_{i_{j}}^{(a)}, 1 \leq i \leq n$, $1 \leq j \leq m^{a-3}$ such that $u \in B_{i_{j}}^{(a)}$. Also, there is a component of the layer $V_{b}$, say $B_{p_{q}}^{(b)}, 1 \leq p \leq n$, $1 \leq q \leq m^{b-3}$ such that $v \in B_{p_{q}}^{(b)}$. In particular, there is a component of the layer $V_{k}$, say $B_{i_{r}}^{(k)}, 1 \leq i \leq n$, $1 \leq r \leq m^{k-3}$ such that, for any vertex $w \in B_{i_{r}}^{(k)}$, we have $d(u, w)=k-a$, and hence, $u$ belongs to a shortest $w-v$ path. 
Case 2.6: let $u, v$ be two distinct vertices in $V(G)-W$ such that $u=\left(v_{1}, 1\right)^{k} \in V_{k}$ and $v \in V_{l}, l \geq 3$. So, there is a component $B_{r_{j_{1}}}^{(l)}$ in the layer $V_{l}, 1 \leq r \leq n$ and $1 \leq j_{1} \leq m^{l-3}$ such that $v \in B_{r_{j_{1}}}^{(l)}$. Thus, there is some vertex in the component $B_{r_{j_{2}}}^{(k)}, 1 \leq j_{2} \leq m^{k-3}$ say $w$ such that $d(w, v)=k-l, d(w, u)>k-l$, and $v$ belongs to a shortest $w-u$ path.

Case 2.7: let $u, v$ be two distinct vertices in $V(G)-W$ such that $u=\left(v_{1_{1}}, 1\right)^{k} \in V_{k}$ and $v \in V_{1}$. So, there is some $i \in V_{1}=\{1,2, \ldots, n\}$ such that $v=i$. If $i=1$, indeed $d(u, v)=k-1$, and then, there is a component $B_{r_{j}}^{(k)}$ in the layer $V_{k}, r \neq 1$ and $1 \leq j \leq m^{k-3}$ such that, for every vertex such as $w$ in the component $B_{r_{j}}^{(k)}$, we have $d(w, v) \geq k, d(w, u) \geq 2 k-1$, and $v$ belongs to a shortest $w-u$ path. Now, let $i \neq 1$; indeed, $d(u, v) \geq k$, and hence, there is a component $B_{i_{j}}^{(k)}$ in the layer $V_{k}$, $1 \leq j \leq m^{k-3}$ such that, for every vertices such as $w$ in the components $B_{i_{j}}^{(k)}$, we have $d(w, v)=k-1$, $d(w, u) \geq 2 k-1$, and $v$ belongs to a shortest $w-u$ path.

Thus, from the abovementioned cases, we can be concluded that the cardinality of minimum strong resolving set of the layer Sun graph $\operatorname{LSG}(n, m, k)$ is $n m^{k-2}-1$.

\subsection{Minimal Doubly Resolving Sets and the Strong Metric} Dimension for the Line Graph of Layer Sun Graph $\operatorname{LSG}(n, m, k)$. Let $G=\operatorname{LSG}(n, m, k)$ be the layer Sun graph which is defined already. Now, let $H$ be a graph with vertex set $V(H)=U_{1} \cup U_{2} \cup \ldots \cup U_{k}$, where $U_{1}, U_{2}, \ldots, U_{k}$ are called the layers of $H$ which is defined as follows:

Let $U_{1}=V\left(C_{n}\right)=\{1,2, \ldots, n\}$ and $U_{2}=\left\{u_{1}, u_{2}, \ldots, u_{n}\right\}$, and for $l \geq 3$, we have

$$
\begin{aligned}
U_{l}= & \left\{D_{1_{1}}^{(l)}, D_{1_{2}}^{(l)}, \ldots, D_{1_{m^{l-3}}}^{(l)} ; D_{2_{1}}^{(l)}, D_{2_{2}}^{(l)}, \ldots,\right. \\
& \left.D_{2_{m^{l-3}}}^{(l)} ; \ldots ; D_{n_{1}}^{(l)}, D_{n_{2}}^{(l)}, \ldots, D_{n_{m^{l-3}}}^{(l)}\right\},
\end{aligned}
$$

and let $D_{\mathrm{ij}}^{(l)}=\left\{\bigcup_{t=1}^{m}\left(u_{\mathrm{ij}}, t\right)^{l}\right\}$ such that every $\left(u_{\mathrm{ij}}, t\right)^{l}$ is a vertex in the layer $U_{l}$ and $D_{\mathrm{ij}}^{(l)} \cong K_{m}$ in the layer $U_{l}$, $1 \leq i \leq n, 1 \leq j \leq m^{l-3}, 1 \leq t \leq m$, where $K_{m}$ is the complete graph on $m$ vertices. Now, suppose that every vertex $i \in\{2,3, \ldots, n\}$ in the cycle $C_{n}$ or the layer $U_{1}$ is adjacent to exactly two vertices in the layer $U_{2}$ say $u_{i}, u_{i-1} \in U_{2}$. In particular, for the vertex 1 in the layer $U_{1}$, we have 1 adjacent to exactly two vertices in the layer $U_{2}$, say $u_{1}, u_{n} \in U_{2}$. Also, every vertex $u_{i}$ in the layer $U_{2}$ is adjacent to exactly $m$ vertices $\left(u_{i 1}, 1\right)^{3},\left(u_{i 1}, 2\right)^{3}, \ldots,\left(u_{i 1}, m\right)^{3} \in D_{i 1}^{(3)} \in U_{3}$, in particular for $l \geq 3$, every vertex $\left(u_{\mathrm{ir}}, t\right)^{l} \in D_{i_{r}}^{(l)} \in U_{l}$ is adjacent to exactly $m$ vertices $\bigcup_{t=1}^{m}\left(u_{\mathrm{ij}}, t\right)^{l+1} \in D_{i_{j}}^{(l+1)} \in U_{l+1}$, and then, the resulting graph is isomorphic with the line graph of the layer Sun graph LSG $(n, m, k)$ with parameters $n$, $m$, and $k$; in fact, $L(G) \cong H$. Note that simply we use refinement of the natural relabelling of the line graph of the graph $\operatorname{LSG}(n, m, k)$. Also, for $l \geq 3$, we recall $D_{i_{j}}^{(l)}$ as the components of $U_{l}, 1 \leq i \leq n, 1 \leq j \leq m^{l-3}$. In particular, we say that two components $D_{i_{j}}^{(l)}, D_{r_{s}}^{(l)} 1 \leq i, r \leq n, 1 \leq j, s \leq m^{l-3}$ are fundamental if $i=r$ and $j \neq s$. It is natural to consider its vertex set of the line graph of the layer Sun graph $\operatorname{LSG}(n, m, k)$ is also as partitioned into $k$ layers. The layers $U_{1}$ and $U_{2}$ consist of the vertices $\{1,2, \ldots, n\}$ and $\left\{u_{1}, u_{2}, \ldots, u_{n}\right\}$, respectively. In particular, each layer $U_{l}$ $(l \geq 3)$ consists of the $\mathrm{nm}^{l-2}$ vertices. Note that, for each vertex $i$ in the layer $U_{1}$ and every vertex $x \in D_{\mathrm{ij}}^{(l)} \in U_{l}, l \geq 3$, $1 \leq j \leq m^{l-3}$, we have $d(i, x)=l-1$. In this section, we consider the problem of determining the cardinality $\psi(L(G))$ of minimal doubly resolving sets of the line graph of the layer Sun graph LSG $(n, m, k)$. We find the minimal doubly resolving set for the line graph of the layer Sun graph $\operatorname{LSG}(n, m, k)$, and in fact, we prove that if $n, k \geq 3$ and $m \geq 2$, then the minimal doubly resolving set of the line graph of the layer Sun graph $\operatorname{LSG}(n, m, k)$ is $\mathrm{nm}^{k-2}-\mathrm{nm}^{k-3}$. Figure 2 shows the line graph of the graph $\operatorname{LSG}(3,3,4)$. Note that simply we use refinement of the natural relabelling of the line graph of the graph $\operatorname{LSG}(3,3,4)$.

Theorem 4. Let $G=\operatorname{LSG}(3,3,4)$ be the layer Sun graph which is defined already. Suppose that $n, m, k$ are integers such that $n, k \geq 3$ and $m \geq 2$. Then, the cardinality of minimum doubly resolving set in the line graph of the graph $G$ is $n m^{k-2}-n m^{k-3}$.

Proof. Let $W$ be an ordered subset of the layer $U_{k}$ in the line graph of the graph $G$ such that

$$
\begin{aligned}
W= & \left\{D_{1_{1}}^{(k)}-\left(u_{1_{1}}, 1\right)^{k}, D_{1_{2}}^{(k)}-\left(u_{1_{2}}, 1\right)^{k}, \ldots, D_{1_{m^{k-3}}}^{(k)}\right. \\
& -\left(u_{1_{m^{k-3}}}, 1\right)^{k} ; \ldots ; D_{n_{1}}^{(k)}-\left(u_{n_{1}}, 1\right)^{k}, D_{n_{2}}^{(k)} \\
& \left.-\left(u_{n_{2}}, 1\right)^{k}, \ldots, D_{n_{m^{k-3}}}^{(k)}-\left(u_{n_{m^{k-3}}}, 1\right)^{k}\right\} .
\end{aligned}
$$

Hence,

$$
\begin{aligned}
V(L(G))-W= & \left\{U_{1}, U_{2}, \ldots, U_{k-1},\left(u_{1_{1}}, 1\right)^{k}, \ldots,\right. \\
& \left.\left(u_{1_{m^{k-3}}}, 1\right)^{k}, \ldots,\left(u_{n_{1}}, 1\right)^{k}, \ldots,\left(u_{n_{m^{k-3}}}, 1\right)^{k}\right\} .
\end{aligned}
$$

We know that $|W|=\mathrm{nm}^{k-2}-\mathrm{nm}^{k-3}$. In a similar way as in Theorem 1, we can show that this subset is a minimal resolving set for the line graph of the graph $G$. We prove that this subset is a doubly resolving set for the line graph of the graph $G$, and hence, $\beta(L(G))=\psi(L(G))$. It is sufficient to prove that, for any two vertices $u$ and $v$ in $L(G)$, there are vertices $\quad x, y \in W \quad$ such that $d(u, x)-d(u, y) \neq$ $d(v, x)-d(v, y)$. Consider two vertices $u$ and $v$ in $L(G)$. Then, we have the following:

Case 1: suppose that both vertices $u$ and $v$ lie in the layer $U_{1}$. Hence, there are $r, s \in\{1,2, \ldots, n\}$ such that $u=r$ and $v=s$. Moreover, we know that the line graph of the graph $G$ has the property that, for each vertex $r$ in 


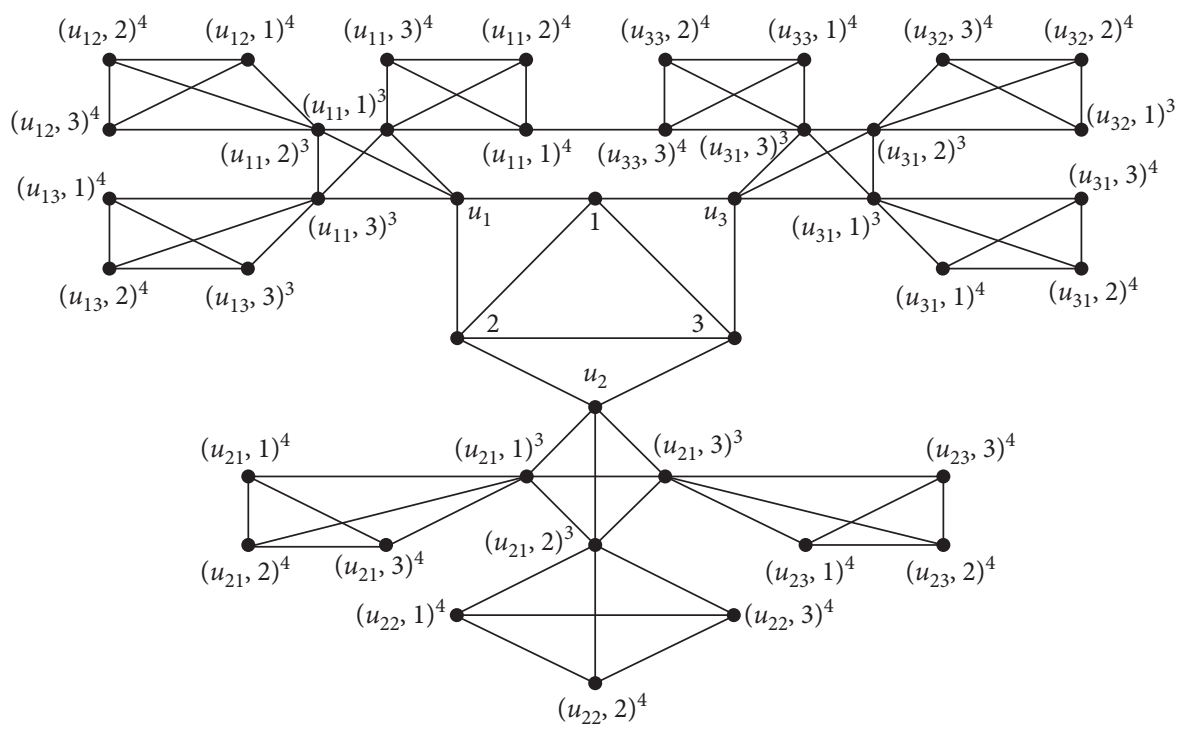

FIgURE 2: The line graph LSG(3,3,4).

the layer $U_{1}$ there is some vertex such as $x=\left(u_{r_{j}}, t\right)^{k}$ in the component $D_{r_{j}}^{(k)}, 1 \leq j \leq m^{k-3}$ in the layer $U_{k}$, at distance $k-1$ from $u$, and in fact, $d(u, x)=k-1$. In the same way, there is some vertex such as $y=\left(u_{s_{j}}, t\right)^{k}$ in the component $D_{s_{j}}^{(k)}$, in the layer $U_{k}$, at distance $k-1$ from $v$. In particular, it is easy to prove that $d(u, x)-$ $d(u, y)<0$ because $d(u, y) \geq k$. Also, $d(v, x)-d(v$, $y)>0$ because $d(v, x) \geq k$.

Case 2: now, suppose that both vertices $u$ and $v$ lie in the layer $U_{2}$. In a similar way as in Case 1 , we can show that there are vertices $x, y \in W$ such that $d(u, x)-d(u, y) \neq d(v, x)-d(v, y)$.

Case 3: suppose that both vertices $u$ and $v$ lie in the layer $U_{l}, l \geq 3$ such that these vertices lie in the one component of the layer $U_{l}$, say $D_{i_{j}}^{(l)}, 1 \leq i \leq n, 1 \leq j \leq m^{l-3}$. In this case, $d(u, v)=1$. Moreover, we know that the line graph of the graph $G$ has the property that, for each vertex $u \in D_{i j}^{(R)}$ in the layer $U_{l}$, there is a component of the layer $U_{k}$, say $D_{i_{r}}^{(k)}, 1 \leq i \leq n, 1 \leq r \leq m^{k-3}$ such that, for every vertex $x \stackrel{i_{r}}{\in} D_{i_{r}}^{(k)}$, we have $d(u, x)=k-l$. In the same way, for the vertex $v \in D_{i_{j}}^{(l)}$ in the layer $V_{l}$, there is a component of the layer $U_{k}$, say $D_{i_{s}}^{(k)}, 1 \leq i \leq n$, $1 \leq s \leq m^{k-3}, r \neq s$ such that, for every vertex $y \in D_{i}^{(k)}$, we have $d(v, y)=k-l$. Thus, $d(u, x)-d(u$, $y) \neq d(v, x)-d(v, y)$ because $d(u, y)=k-l+1$ and $d(v, x)=k-l+1$.

Case 4: suppose that both vertices $u$ and $v$ lie in the layer $U_{l}, l \geq 3$ such that these vertices lie in the two distinct components of the layer $U_{l}$. We can assume without loss of generality that $u \in D_{p_{j_{1}}}^{(l)}$ and $v \in D_{q_{j_{2}}}^{(l)}, 1 \leq p, q \leq n$, and $1 \leq j_{1}, j_{2} \leq m^{l-3}$. Moreover, we know that the line graph of the graph $G$ has the property that, for each vertex $u \in D_{p_{j_{1}}}^{(l)}$ in the layer $U_{l}$, there is a component of the layer $U_{k}$, say $D_{p_{r}}^{(k)}, 1 \leq r \leq m^{k-3}$ such that, for every vertex $x \in D_{p_{r}}^{(k)}$, we have $d(u, x)=k-l$. In the same way, for the vertex $v \in D_{q_{j_{2}}}^{(l)}$ in the layer $V_{l}$, there is a component of the layer $U_{k}^{q_{j_{2}}}$, say $D_{q_{s}}^{(k)}, 1 \leq s \leq m^{k-3}$, such that, for every vertex $y \in D_{q_{s}}^{(k)}$, we have $d(v, y)=k-l$. In the following, let two components $D_{p_{j_{1}}}^{(l)}$, and $D_{q_{i_{2}}}^{(l)}$ be fundamental; indeed, $p=q$. Hence, $d(u, v)=2 l^{q_{2}}-5$, $d(u, y)=d(v, x)=k+l-5$. Thus, $d(u, x)-d(u, y) \neq$ $d(v, x)-d(v, y)$. Now, let two components $D_{p_{j_{1}}}^{(l)}$ and $D_{q_{j_{2}}}^{(l)}$ not be fundamental; indeed, $p \neq q$. Hence, $d(u, v)=2 l-4+d_{U_{2}}\left(u_{p}, u_{q}\right)$ and $d(u, y)=d(v, x)=$ $k+l-4+d_{U_{2}}\left(u_{p}, u_{q}\right)$. Thus, $d(u, x)-d(u, y) \neq$ $d(v, x)-d(v, y)$.

Case 5: suppose that vertices $u$ and $v$ lie in distinct layers $U_{a}, U_{b}$, respectively. Note that if $a=1$ and $b=2$, $a=1$ and $b>2$, or $a=2$ and $b>2$, there is nothing to do. Now, let $3 \leq a<b$. Hence, there is a component of the layer $U_{a}$, say $D_{i_{j}}^{(a)}, 1 \leq i \leq n, 1 \leq j \leq m^{a-3}$ such that $u \in D_{i_{j}}^{(a)}$. Also, there is a component of the layer $U_{b}$, say $D_{p_{q}}^{(b)}, \quad 1 \leq p \leq n, \quad 1 \leq q \leq m^{b-3}$ such that $v \in D_{p_{q}}^{(b)}$. In particular, there is a component of the layer $U_{k}$, say $D_{i_{r}}^{(k)}, 1 \leq i \leq n, 1 \leq r \leq m^{k-3}$ such that, for any vertex $x \in D_{i_{r}}^{(k)}$, we have $d(u, x)=k-a$. Now, let $i=p$; if we consider $y \in D_{z_{s}}^{(k)}, z \neq i, 1 \leq z \leq n$, and $1 \leq s \leq m^{k-3}$, then we have $d(u, x)-d(u, y) \neq d(v, x)-d(v, y)$ because $d(u, y)=k+a-4+d_{U_{2}}\left(u_{i}, u_{z}\right), \quad d(v, y)=k+b-4+$ $d_{U_{2}}\left(u_{i}, u_{z}\right)$, and $d(u, x) \neq d(v, x)$. Note that if $i \neq p$, then there is a component of the layer $U_{k}$, say $D_{p_{s}}^{(k)}$, $1 \leq p \leq n, \quad 1 \leq s \leq m^{k-3}$ such that, for any vertex $y \in D_{p_{j}}^{(k)}$, we have $d(v, y)=k-b$, and then, we have $d(u, x)-d(u, y) \neq d(v, x)-d(v, y)$.

Thus, from the abovementioned cases, we conclude that the cardinality of minimum doubly resolving set in the line graph of the graph $G$ is $\mathrm{nm}^{k-2}-\mathrm{nm}^{k-3}$. 
Theorem 5. Let $G=\operatorname{LSG}(n, m, k)$ be the layer Sun graph which is defined already. Suppose that $n, m, k$ are integers such that $n, k \geq 3$ and $m \geq 2$. Then, the strong metric dimension in the line graph of the graph $G$ is $n m^{k-2}-1$.

Proof. In a similar way as in the proof of Theorem 3, we can show that the subset

$$
W=\left\{D_{1_{1}}^{(k)}-\left(v_{1_{1}}, 1\right)^{k}, D_{1_{2}}^{(k)}, \ldots, D_{1_{m^{k-3}}}^{(k)}, \ldots, D_{n_{1}}^{(k)}, D_{n_{2}}^{(k)}, \ldots, D_{n_{m^{k-3}}}^{(k)}\right\}
$$

of vertices in the line graph of the graph $G$ is a minimal resolving set of $L(G)$.

\section{Conclusions}

In this paper, we have constructed a layer Sun graph LSG $(n, m, k)$, discussed this graph, and computed the minimum cardinality of the doubly resolving set and strong resolving set of layer Sun graph $\operatorname{LSG}(n, m, k)$ and the line graph of the layer Sun graph LSG $(n, m, k)$. We deduce that, by this way, we can construct a layer jellyfish graph JFG $(n, m, k)$, of order $n+\sum_{r=1}^{k-1} \mathrm{~nm}^{r}$, where the jellyfish graph is JFG $(n, m)$, which is defined in [5], and by a similar way, we can obtain and compute the minimum cardinality of doubly resolving set and strong resolving set of layer jellyfish graph $\operatorname{JFG}(n, m, k)$ and the line graph of the layer jellyfish graph $\mathrm{JFG}(n, m, k)$.

\section{Data Availability}

No data were used to support this study.

\section{Conflicts of Interest}

The authors declare that there are no conflicts of interest regarding the publication of this paper.

\section{Acknowledgments}

This work was partially supported by the Project of Anhui Jianzhu University under grant nos. 2016QD116 and $2017 \mathrm{dc} 03$.

\section{References}

[1] C. Godsil and G. Royle, Algebraic Graph Theory, Springer, New York, NY, USA, 2001.

[2] J. Cáceres, C. Hernando, M. Mora et al., "On the metric dimension of Cartesian products of graphs," SIAM Journal on Discrete Mathematics, vol. 21, no. 2, pp. 423-441, 2007.

[3] A. Sebö and E. Tannier, "On metric generators of graphs," Mathematics of Operations Research, vol. 29, no. 2, pp. 383393, 2004.

[4] O. R. Oellermann and J. Peters-Fransen, "The strong metric dimension of graphs and digraphs," Discrete Applied Mathematics, vol. 155, no. 3, pp. 356-364, 2007.

[5] J.-B. Liu, A. Zafari, and H. Zarei, "Metric dimension, minimal doubly resolving sets, and the strong metric dimension for jellyfish graph and cocktail party graph," Complexity, vol. 2020, Article ID 9407456, 7 pages, 2020.
[6] G. Chartrand, L. Eroh, M. A. Johnson, and O. R. Oellermann, "Resolvability in graphs and the metric dimension of a graph," Discrete Applied Mathematics, vol. 105, no. 1-3, pp. 99-113, 2000.

[7] F. Harary and R. A. Melter, "On the metric dimension of a graph," Combinatoria, vol. 2, pp. 191-195, 1976.

[8] J. Kratica, M. Čangalović, and V. Kovačević-Vujčić, "Computing minimal doubly resolving sets of graphs," Computers \& Operations Research, vol. 36, no. 7, pp. 2149-2159, 2009.

[9] J. Kratica, V. Kovacevic-Vujcic, M. Stojanovic, and M. Stojanović, "Minimal doubly resolving sets and the strong metric dimension of Hamming graphs," Applicable Analysis and Discrete Mathematics, vol. 6, no. 1, pp. 63-71, 2012.

[10] J. Kratica, V. Kovačević-Vujčić, M. Čangalović, and M. Stojanović, "Minimal doubly resolving sets and the strong metric dimension of some convex polytopes," Applied Mathematics and Computation, vol. 218, no. 19, pp. 97909801, 2012.

[11] J. Kratica, V. Kovacevic-Vujcic, M. Mladenovic, and N. Mladenović, "Strong metric dimension: a survey," Yugoslav Journal of Operations Research, vol. 24, no. 2, pp. 187-198, 2014.

[12] J.-B. Liu, M. F. Nadeem, H. M. A. Siddiqui, and W. Nazir, "Computing metric dimension of certain families of toeplitz graphs," IEEE Access, vol. 7, pp. 126734-126741, 2019.

[13] X. Ma and Y. She, "The metric dimension of the enhanced power graph of a finite group," Journal of Algebra and Its Applications, vol. 19, pp. 1-14, 2019.

[14] S. Pirzada, M. Aijaz, and S. P. Redmond, "On upper dimension of graphs and their bases sets," Discrete Mathematics Letters, vol. 3, pp. 37-43, 2020.

[15] Z. Shao, S. M. Sheikholeslami, P. Wu, and J.-B. Liu, "The metric dimension of some generalized petersen graphs," Discrete Dynamics in Nature and Society, vol. 2018, Article ID 4531958, 10 pages, 2018.

[16] P. J. Slater, "Leaves of trees," in Proceedings of the 6th Southeastern Conference on Combinatorics, Graph theory and Computing, pp. 549-559, Boca Raton, FL, USA, February 1975. 